\title{
A Detail Process for CAD Based Breast Cancer Detection
}

\author{
Priyanka Banerjee ${ }^{1}$ and Samir Kumar Bandyopadhyay ${ }^{2, *}$
}

${ }^{1}$ Department of Computer Science, The Bhawanipur Education Society College, Kolkata, India

${ }^{2}$ Academic Advisor, The Bhawanipur Education Society College, Kolkata, India

\begin{abstract}
Breast cancer is known to cause high mortality unless detected in time. Early detection during the onset of the disease can prevent mortality. Early detection can prevent the spreading of the disease thus providing a healthy life to senior citizens. Mammographic screening and surgical biopsy will yield huge number of images to be deciphered by radiologists and pathologists respectively. MIAS dataset is sufficiently large to conduct experimental analysis. Moreover, the dataset contains 322 mammogram images of different size, shape and morphology. This paper discussed about breast cancer detection and diagnosis process. (Word count -91 words).
\end{abstract}

Keywords: Breast cancer, mammography, risk factors, Pectoral Muscle.

\section{INTRODUCTION}

Computer Aided Diagnostics (CAD) requires medical Image Processing, a multi-disciplinary science, for development of algorithms based on medical images. The digital mammogram screening technique can only detect the abnormalities like presence of tumours or cysts. According to the cancer experts, some morphological and anatomical signs in mammogram are significant for future risk determination of breast cancer development. The detection method is based on mammogram analysis. The input for the process is raw digital mammogram images and output is the identification of abnormalities if present. The detection method can be divided into three sequential distinct subsections namely preparation, pre-processing, and abnormality identification.

The preparation phase is needed in order to improve the image quality and make the segmentation results more accurate. It is done by two processes, as given below, along with noise removal using Gaussian smoothening.

\section{Artefact Removal Algorithm \\ 2. Image Orientation Algorithm}

The pre-processing phase consists of mammogram image registration and edge detection, pectoral muscle suppression, contour determination and anatomical segmentation. The pre-processing methods consist of:

1. Homogeneity Enhancement

2. Edge Detection using standard method

*Address correspondence to this author at Academic Advisor, The Bhawanipur Education Society College, Kolkata, India; E-mail: 1954samir@gmail.com
3. Pectoral Muscle Suppression

4. Breast Contour Detection

5. Anatomical Segmentation

The abnormality identification detects abnormalities, if present, in the mammogram. Through identification and localization of mass created in the breast.

Mammogram screening also includes future risk determination. There are three proven risk markers that has been considered for future risk determination namely, asymmetry analysis, volume calculation and parenchymal density estimation. The preparation and pre- processing steps are pre-requisite for the implementation of these risks. Normally the following processes have been done for the purpose:

1. Bilateral Breast Asymmetry Detection

\section{Breast Volume Calculation}

\section{Density Estimation and Classification}

Different size, shape and types i.e. fatty, fattyglandular and dense-glandular mammograms were evaluated individually because of their different contrast level and intensity properties.

The objective of the all methods is not to replace the radiologist or pathologist but to assist them for early and efficient diagnosis of human breast cancer. It is hoped that the processes are described here may help in other image processing problems specifically in the field of medical image processing.

\section{LITERATURE REVIEW}

The different technologies which are used to view the human body in order to diagnose, monitor or treat 
medical conditions are referred to as Medical Imaging. Specific technologies are used to investigate and analyze different information about the explicit area of the body being studied or treated, related to possible disease, injury or the helpful for medical treatment. There are several medical imaging technologies that are used nowadays like X-ray, Ultrasonography (USG), Computer Tomography (CT) and Magnetic Resonance Imaging (MRI) etc. In breast cancer diagnosis several imaging technologies are used including Mammography, Ultrasonography and Magnetic Resonance Imaging. Mammogram is used to identify breast cancer. Several researches have shown that early detection with mammography saves lives and increases treatment options [1-3]. One of the main advantages of mammography is the comparative cost effectiveness and availability.

Recent studies reveal that majority of clinical tests including digital mammography and biopsies performed are benign, resulting in wastage of valuable time of medical practitioners and at the same time increasing the possibility of false detection [4-6]. The efficient CAD system can screen the benign cases and assist the experts in terms of qualitative and quantitative precision [7].

The common architecture of a CAD system for digital mammogram includes image preparation, preprocessing, features extraction and detection. The basic building blocks of those processes are image preparation including orientation, artefact removal and noise reduction, image registration, image enhancement, segmentation and detection. The process of segmentation is most important topic of CAD analysis. In mammographic image analysis, segmentation is related with the pectoral muscle suppression, edge detection, determination of Region of Interest (ROI), anatomical segmentation, segmentation of abnormalities like mass and microcalcification.

\section{PROCESS FOR BREAST CANCER DETECTION}

The breast is prone to various benign and malignant conditions. The most common benign conditions are puerperal mastitis, fibrocystic breast changes and mastalgia. The latter part is very serious in nature that is Breast Cancer. Breast cancer, a malignant tumour developed from breast cells is considered to be one of the major causes for the increase in mortality among women, especially in developed and developing countries. More specifically, breast cancer is the second most common type of cancer and the fifth most common cause of cancer-related death. So, it continues to be a significant public health problem in the world.

Cancer is a type of diseases in which cells in the body to change and grow out of control. Formation of lump or mass called a tumour, and are named after the part of the body where the tumour originates. Breast cancer begins in breast tissue. After that it may spread in other cells of the human body also.

Most masses are benign, that is, they are not cancerous, do not grow uncontrollably or spread and are not life-threatening. Some breast cancers are called in situ because they are confined within the ducts (ductal carcinoma in situ) or lobules (lobular carcinoma in situ) of the breast. Nearly all cancers at this stage can be cured. Invasive cancers start in the lobules or ducts of the breast but have broken through the duct or glandular walls to invade the surrounding tissue of the breast.

Breast cancer stages range from 0 to IV i.e. normal to advance stage. The invasive breast cancer is strongly influenced by the stage of the disease and depends on the extent or spread of the cancer. Early and efficient diagnosis is the most effective way for treatment and to reduce mortality. The breast cancer diagnosis process is basically two folds. The screening process is simply used to find abnormalities in the breast. The conclusion reached through process of analyzing histopathological slide with present technologies and procedures like biopsy to determine whether a tumour is malignant or benign.

Breast cancer screening is a professional medical examination performed to check women's breasts for abnormalities such as calcification, tumours and cysts, and identify where they exist. For examining the breast cancer, Ultrasound imaging, which uses a band of high frequency sound waves to probe the breast, Magnetic Resonance Imaging (MRI), which probes the breast using powerful magnetic fields and Mammography, which is essentially producing X-ray photographs of the breast.

Digital mammography is a recent technique for recording $\mathrm{x}$-ray images in digital code instead of on $\mathrm{x}$ ray film, as with conventional mammography. Digital mammography has some advantages over conventional mammography. The images are displayed on a computer monitor and can be enhanced before 
they are printed on film. Since the images are stored and retrieved electronically so it helps consultations with other mammography specialists easier. Digital Mammography has been proved to be the most effective and reliable screening method for early breast cancer detection.

Imaging techniques play an important role in helping to perform breast biopsies, especially of abnormal areas that can be identified and located by a mammogram. Open surgical biopsy is the gold standard as compared to other methods of breast biopsies.

The general methods of image preparation can be divided into three parts: orientation, artefact removal and de- noising. The mammogram images of breasts are not homogeneously oriented in common projection of mammogram in MLO view. The images contain left and right orientation of breast. For automatic processing it is important to make them homogeneous to reduce the additional algorithmic complexity. Artefacts are identification marks that are used on the mammogram to identify the patient and related data that may be required for identification of the mammogram. These markings provide high intensity regions on the mammogram and are inconsequential to the investigation of abnormalities within the mammogram. As per the characteristic feature of mammogram, breast region is represented by high intensity pixels, where as background consist of chest wall and skin to air are represented by ideally zero intensity or by very low intensity. The artefacts and other irrelevant object are present in background, more specifically in the skin to air part of mammogram image. These artefacts are removed by the searching of the region with a threshold value. Noises also make false results or negative detection. Gaussian filter is used to remove such noise. The Gaussian smoothing operator is a 2-D convolution operator that is used to 'blur' images and remove noise.

Mammogram registration and edge detection plays a critical step in automatic detection of breast anomalies. Combining anatomical features and intensity-based homogeneity features are used for image registration techniques. First Value, Min-Max, Mean, Median and Mode are applied to obtain the central tendencies of image intensities to enhance homogeneity of image.

The homogeneity process is followed by detection of edges in an image. This is a very important step towards understanding image features. When images are divided into areas corresponding to different objects edge detection is extensively used in image segmentation. This can be used specifically for enhancing the tumour area in mammographic images. Different existing methods are available for edge detection.

The pectoral muscle, a non-breast region in mammograms, acts like an additional complexity in automated system. The initial step involves defining a rectangle to isolate the pectoral muscle from the region of interest (ROI) and the next is to suppress the pectoral muscle using identification of region.

The breast contour is important feature within the breast region. So it is required to extract breast region and breast contour that allow the search for abnormalities. Boundary detection is used on mammographic images for breast detection so that any abnormalities can be detected.

The Anatomical Segmentation of Breast ROI (ASB) method is applied to differentiate various regions within the breast. There are significant research efforts have gone into developing computerized methods to detect abnormal masses with different intensity value of various types and classify them.

An abnormality such as a mass, tumours or calcifications may be present within the breast has distinctly higher intensity values than the normal tissues of the breast. So, it is needed to categorize all the obtained closed structures on basis of their intensity values. The outputs of all the process so far described are given Figures 1 to 17 for demonstration purpose.

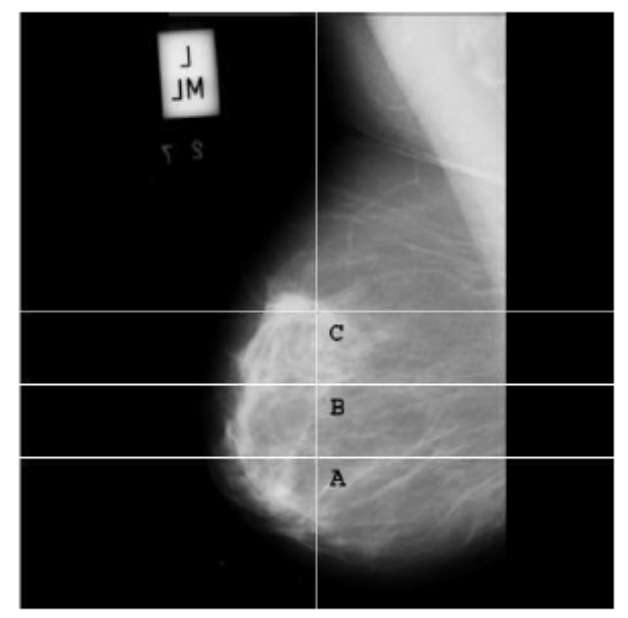

Figure 1: Original Mammogram showing the preferred pixel location ( $A, B$ and $C)$. 

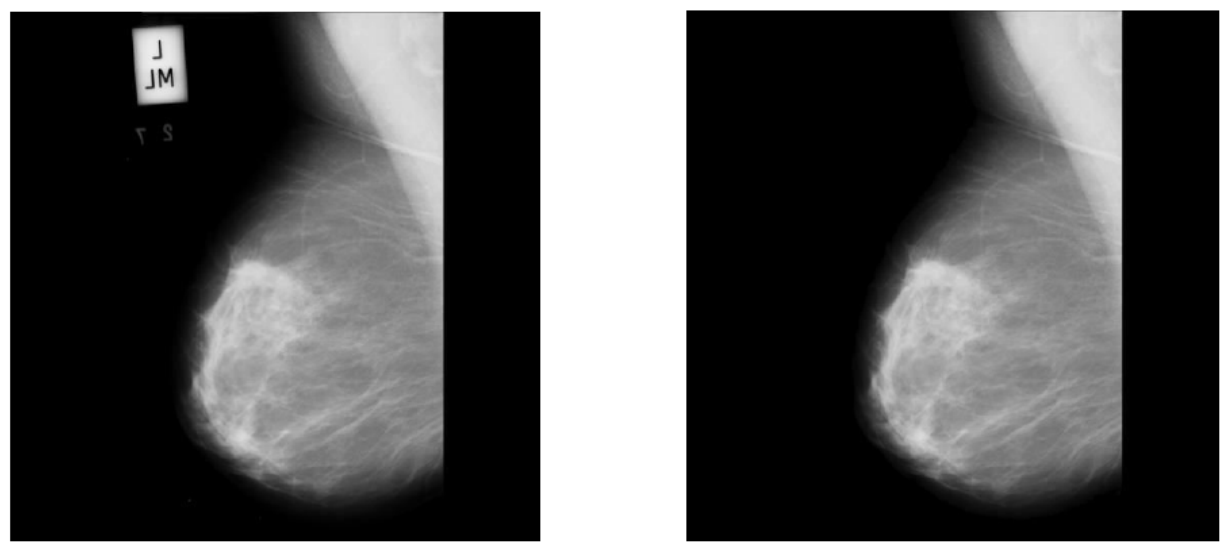

Figure 2: Original Mammogram and Mammogram without artefact.
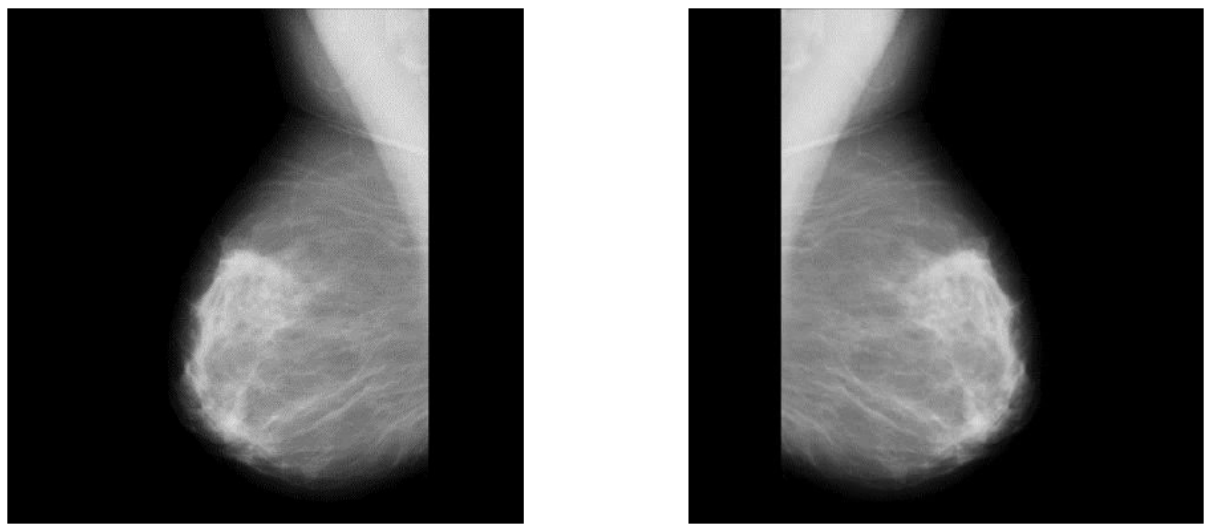

Figure 3: Right Mammogram and Mammogram after Flipping $180^{\circ}$.
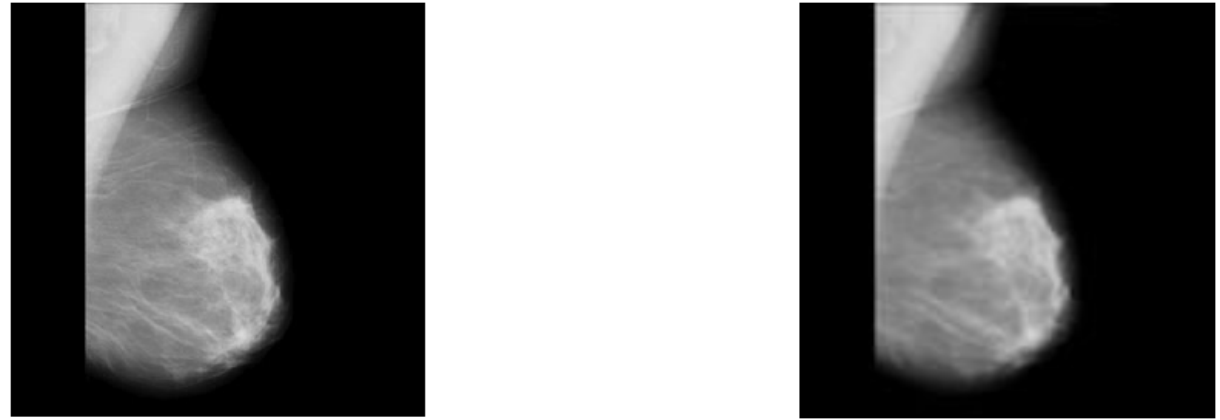

Figure 4: Mammogram before and after Gaussian Smoothening for noise removal.
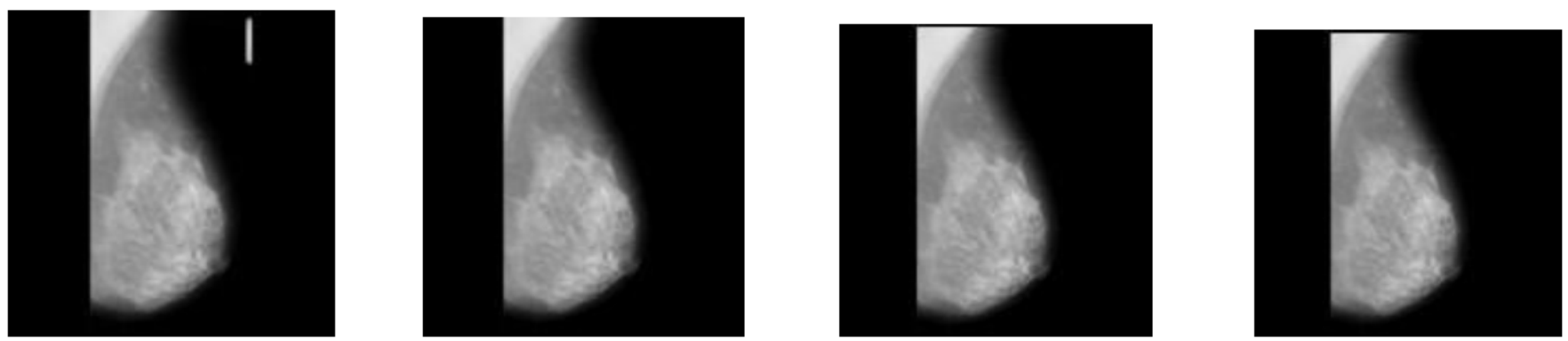

Figure 5: Images showing Original Mammogram followed by image after Artefacts Removable, Flipping (no change) and Noise Removable (Images from left to right). 


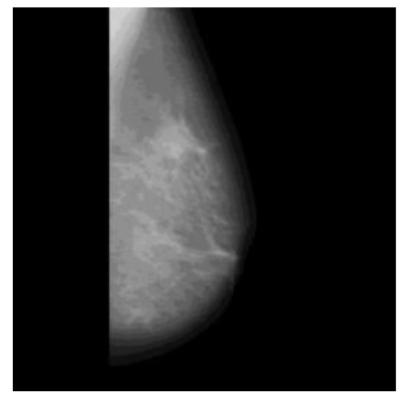

(a)

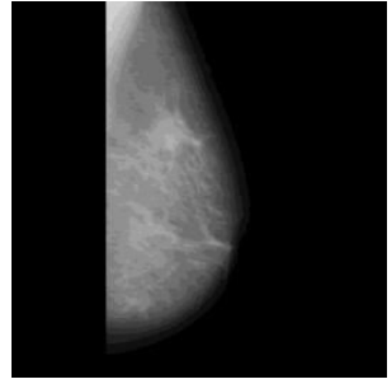

(d)

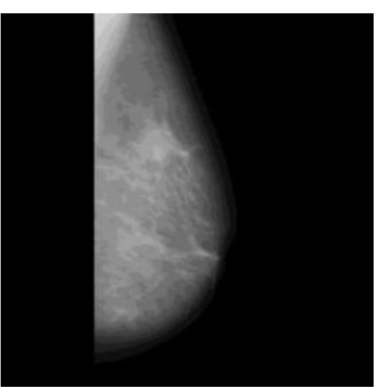

(b)

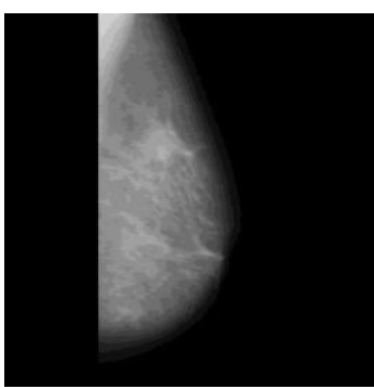

(c)

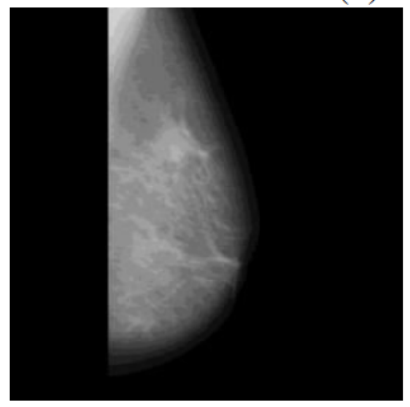

(e)

Figure 6: Mammogram (MIAS 092.L) after DCHEA using (a) First Value Method, (b) Min- Max Method, (c) Mean Value Method, (d) Median Value Method and (e) Mode Value Method.

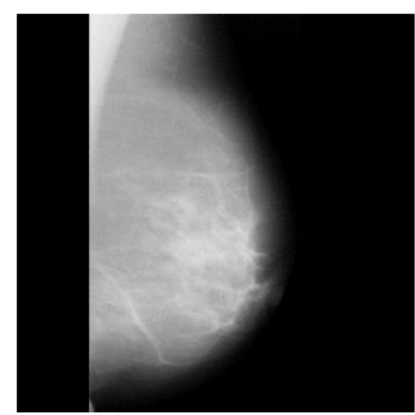

(a)

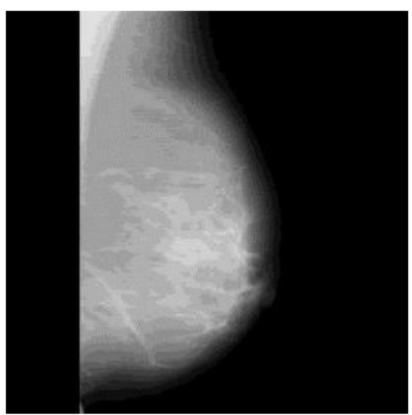

(b)

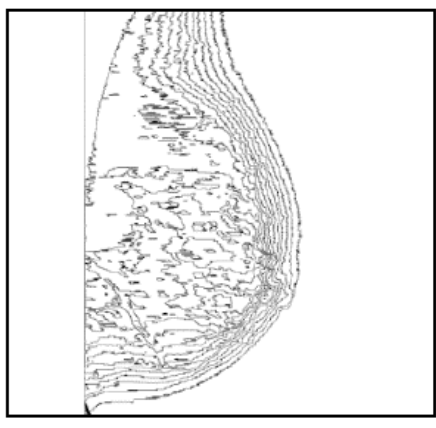

(c)

Figure 7: Original Mammogram Image along with image after Enhancement.

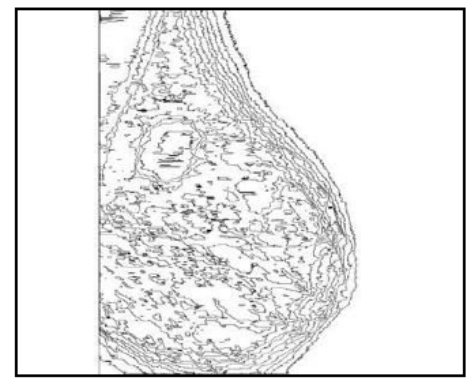

(a)

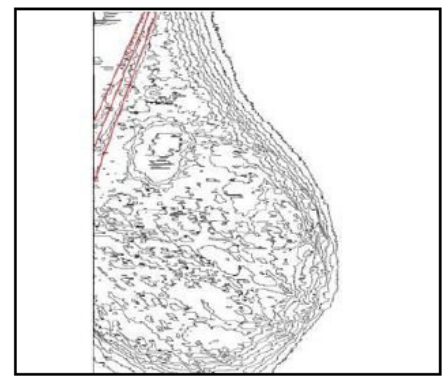

(b)

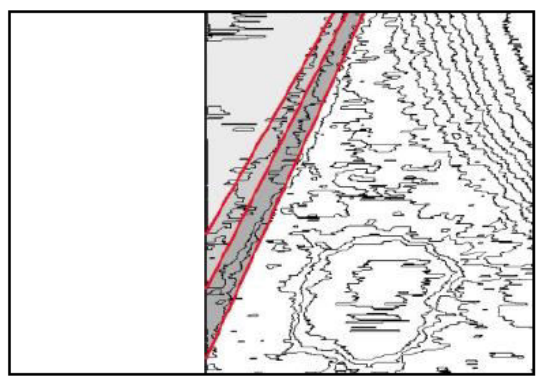

(c)

Figure 8: (a) The Edge Map of Mammogram, (b) Showing the layers of Pectoral Muscle and (c) showing inverted triangles marked by different grey shades. 

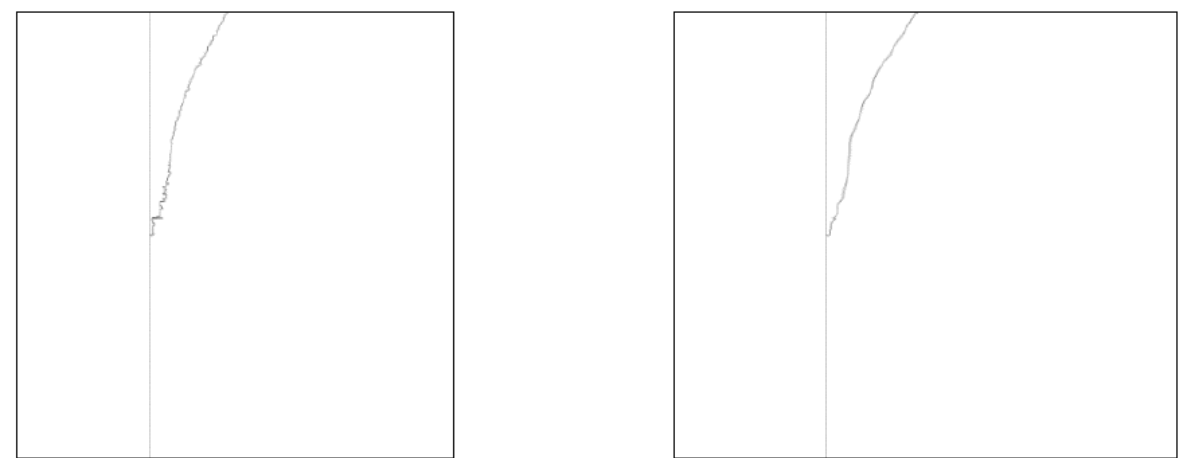

Figure 9: Detected Pectoral Boundary and Smooth Boundary.
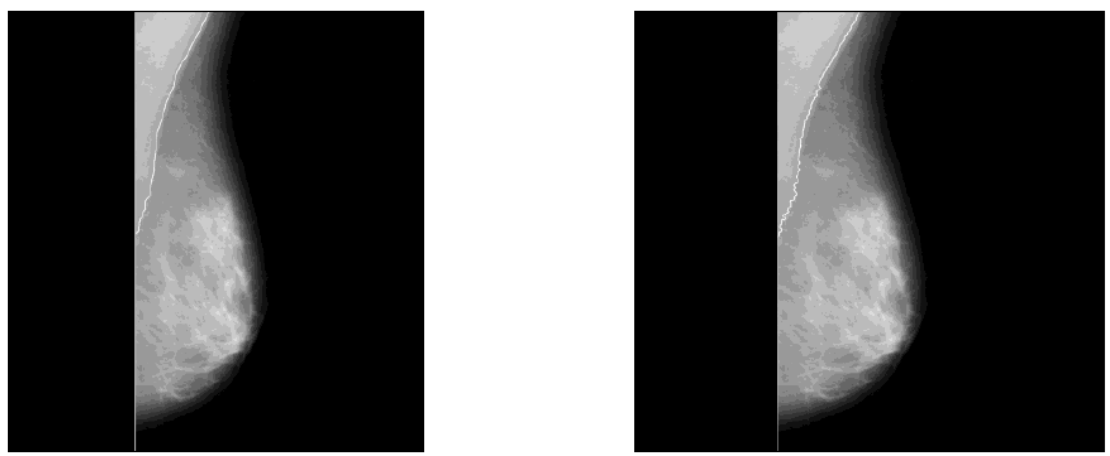

Figure 10: Detected Pectoral Boundary and Ground Truth superimposed on Original Mammogram.
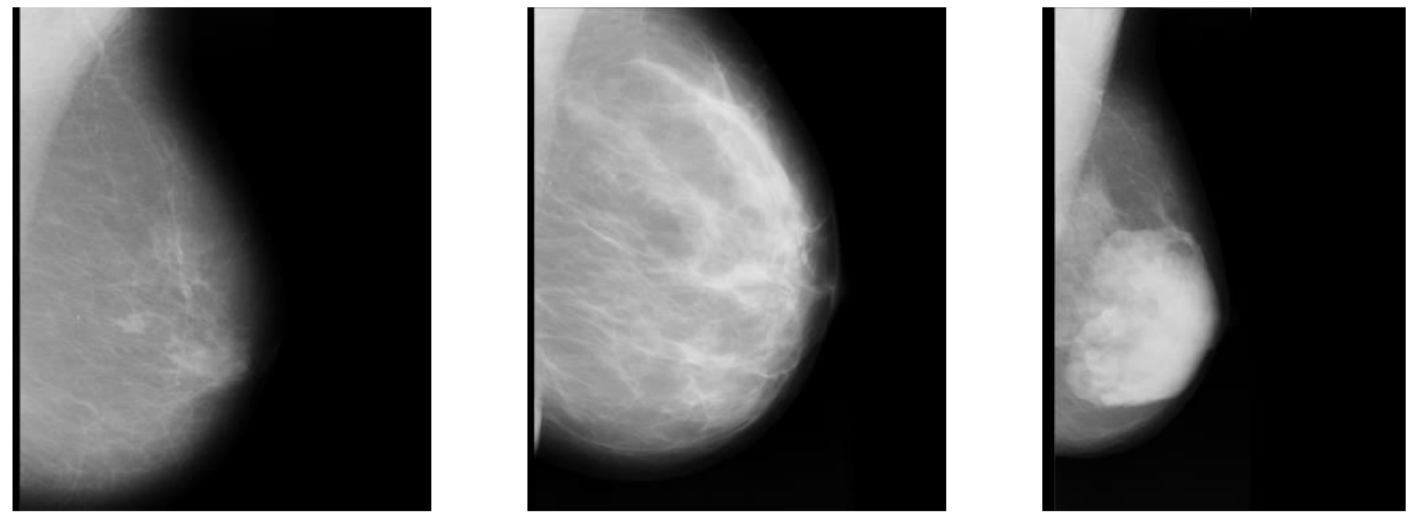

Figure 11: The Original Sample of Mammograms (Fatty, Fatty-glandular, Dense-glandular from left to right).
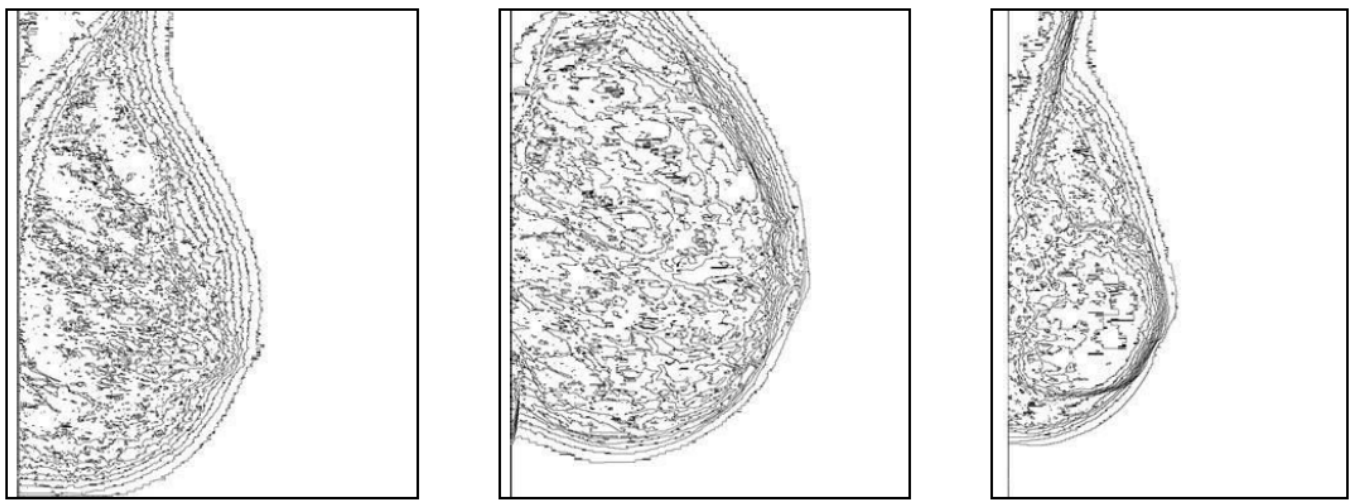

Figure 12: The Edge Map of Mammograms using EDA (Fatty, Fatty-glandular, Dense-glandular from left to right). 

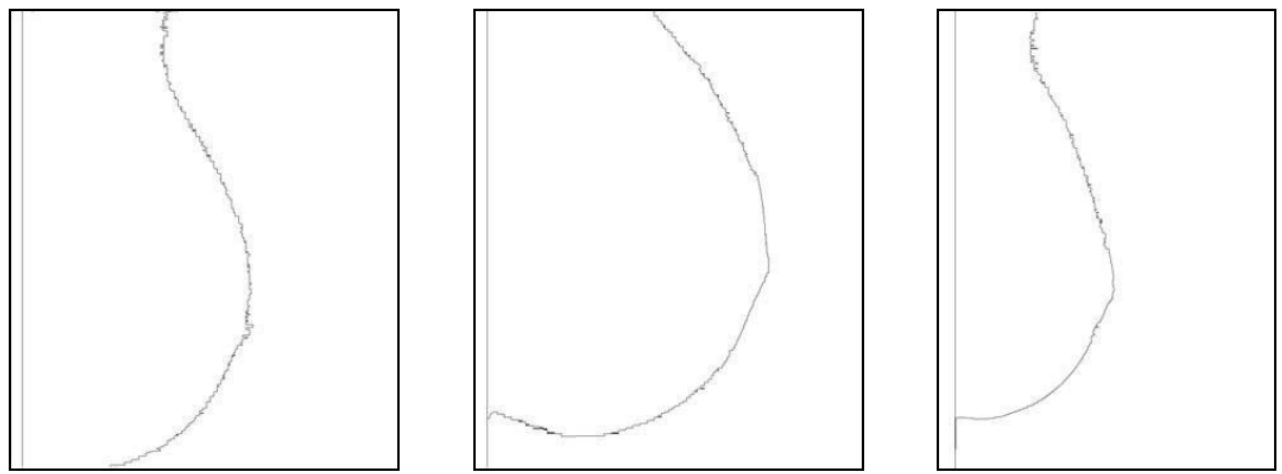

Figure 13: Breast Boundary of Mammogram using BBDA (Fatty, Fatty-glandular, Dense-glandular from left to right).
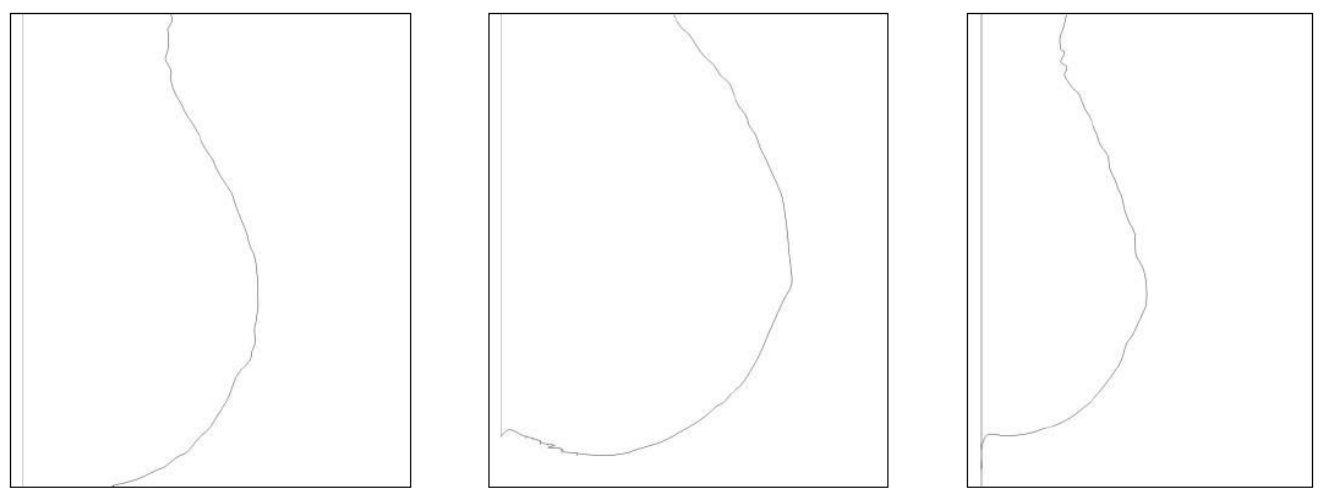

Figure 14: Smooth and Enhanced Breast Boundary (Fatty, Fatty-glandular, Dense-glandular from left to right).

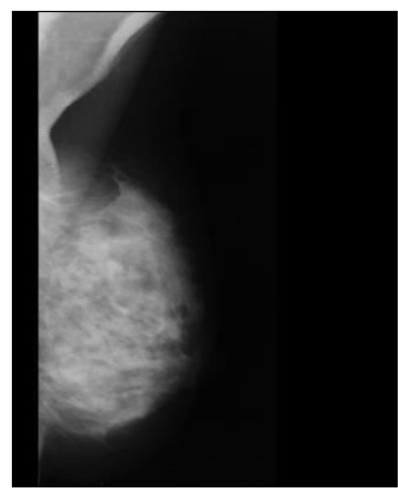

(a)

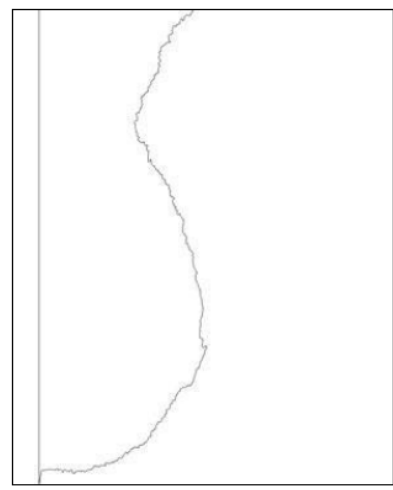

(b)

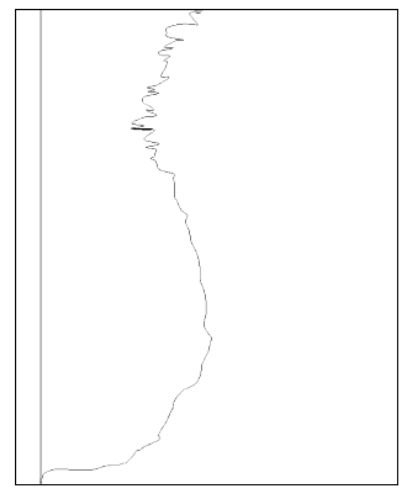

(c)

Figure 15: (a) Original Image, (b) Ground Truth and (c) Boundary Retrieved by the Proposed Method from original image.

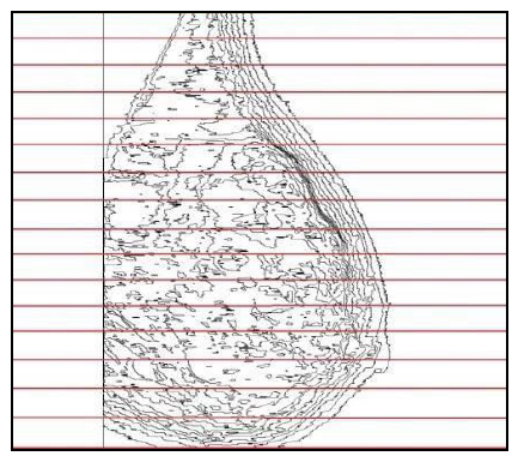

Figure 16: Extract the Anatomical Segmentation from Edge.

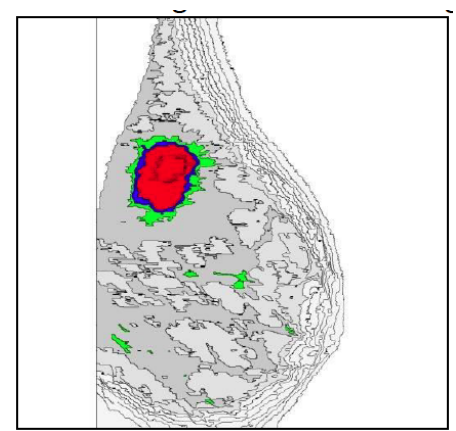

Figure 17: Highlighted Regions with Abnormal Masses Marked Red Colour in Mammogram Image. 


\section{CONCLUSIONS}

Computer aided detection and computer aided diagnosis are one of the most important field of research due to its proven competence and accuracy. Significant development of mammogram based computer aided detection using image processing techniques has been reported by different research articles. This paper has given outline of entire process for detection of mass in breast that are required to detect breast cancer.

\section{REFERENCES}

[1] Albregtsen F, Nielsen B, Danielsen HE. Adaptive gray level run length features from class distance matrices. Proceedings - International Conference on Pattern Recognition 2000; 15(3): 738-741. https://doi.org/10.1109/icpr.2000.903650

[2] American Cancer Society. Breast Cancer Facts \& Figures 2019-2020. American Cancer Society 2019; pp. 1-44. https://doi.org/10.1007/978-1-4614-6439-6 151-2

[3] Chandrasekhar R, Attikiouzel Y. Segmentation of the pectoral muscle edge on mammograms by tunable parametric edge detection. Advances in Signal Processing and Computer Technologies 2001; pp. 55-60. Retrieved from https://pdfs.semanticscholar.org/dec0/c39a34f1a4b8879cd9f c97a69cc9f2c4e65f.pdf? ga=2.263117132.1774612510.157 7001857-1752445732.1577001857

[4] Bahlmann C, Patel A, Johnson J, Ni J, Chekkoury A, Khurd $\mathrm{P}$, Weinstein R. Automated detection of diagnostically relevant regions in H\&E stained digital pathology slides. Medical Imaging 2012: Computer-Aided Diagnosis 2012; 8315: 831504. https://doi.org/10.1117/12.912484

[5] Ball JE, Bruce LM. Digital Mammographic Computer Aided Diagnosis (CAD) using Adaptive Level Set Segmentation. Proceedings of the 29th Annual International Conference of the IEEE EMBS Cité Internationale 2007; pp. 4973-4978. https://doi.org/10.1109/IEMBS.2007.4353457

[6] Ali MA, Czene K, Eriksson L, Hall P, Humphreys K. Breast tissue organisation and its association with breast cancer risk. Breast Cancer Research 2017; 19(1): 1-13. https://doi.org/10.1186/s13058-017-0894-6

[7] Oliveira HCR, de Mencattini A, Casti P, Martinelli E, Natale $C$ di Catani JH, Vieira MAC. Reduction of false-positives in a CAD scheme for automated detection of architectural distortion in digital mammography. SPIE Medical Imaging 2018. Retrieved from https://www.spiedigitallibrary. org/conference-proceedings-of-spie/10575/105752P/ Reduction-of-false-positives-in-a-CAD-scheme-forautomated/10.1117/12.2293388.short?tab=ArticleLink

DOI: http://dx.doi.org/10.30683/1929-2279.2019.08.03

(C) 2019 Banerjee and Bandyopadhyay; Licensee Neoplasia Research.

This is an open access article licensed under the terms of the Creative Commons Attribution Non-Commercial License (http://creativecommons.org/licenses/by-nc/3.0/) which permits unrestricted, non-commercial use, distribution and reproduction in any medium, provided the work is properly cited. 